\title{
Filigrane
}

Écoutes psychanalytiques

\section{Violences meurtrielles}

\section{Robert C. Colin}

Volume 25, numéro 2, 2016

Le sujet de la violence

URI : https://id.erudit.org/iderudit/1039650ar

DOI : https://doi.org/10.7202/1039650ar

Aller au sommaire du numéro

\section{Éditeur(s)}

Revue Santé mentale au Québec

\section{ISSN}

1192-1412 (imprimé)

1911-4656 (numérique)

Découvrir la revue

\section{Citer cet article}

Colin, R. C. (2016). Violences meurtrielles. Filigrane, 25(2), 103-119. https://doi.org/10.7202/1039650ar

\section{Résumé de l'article}

La violence est partout présente. Elle est tantôt expression et affirmation de la vie et exerce alors un effet structurant sur la psyché ; tantôt elle est destructrice, meurtrière ou potentiellement désubjectivante. Si la première est confinée dans un cadre défini que le droit est censé protéger et que la loi symbolique devrait circonscrire, la seconde est de nature transgressive et emprunte des formes multiples, non nécessairement spectaculaires. Elle est " meurtrielle » dans le sens où elle est un équivalent fantasmatique de meurtre. Cet article présente cinq exemples cliniques dans des registres différents : violence sans nom, violence extrême, violence défensive, violence régressive et violence culturelle.
Ce document est protégé par la loi sur le droit d'auteur. L'utilisation des services d’Érudit (y compris la reproduction) est assujettie à sa politique d'utilisation que vous pouvez consulter en ligne.

https://apropos.erudit.org/fr/usagers/politique-dutilisation/ 


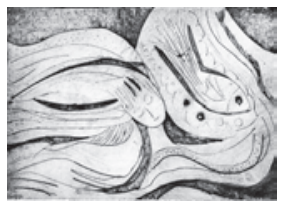

\title{
Violences meurtrielles
}

\author{
Robert C. Colin
}

RÉSUMÉ: La violence est partout présente. Elle est tantôt expression et affirmation de la vie et exerce alors un effet structurant sur la psyché; tantôt elle est destructrice, meurtrière ou potentiellement désubjectivante. Si la première est confinée dans un cadre défini que le droit est censé protéger et que la loi symbolique devrait circonscrire, la seconde est de nature transgressive et emprunte des formes multiples, non nécessairement spectaculaires. Elle est «meurtrielle» dans le sens où elle est un équivalent fantasmatique de meurtre. Cet article présente cinq exemples cliniques dans des registres différents: violence sans nom, violence extrême, violence défensive, violence régressive et violence culturelle.

Mots clés: Violence, transgression, régression

ontribuer à ce numéro de la revue Filigrane me réjouit et me permet d'approfondir, autour du thème de la violence, des recherches qui m'ont occupé pendant plusieurs années. Au moment de m'y consacrer, le thème m'apparaît vaste et insaisissable. La violence semble partout présente et ce n'est pas la plus spectaculaire qui, dans la pratique psychanalytique, paraît la plus révoltante. Je ne suis pas gêné par la violence légitime, tournée du côté de la vie, structurante pour la psyché, si revigorante, mais je le suis par l'omniprésence de la violence que Piera Aulagnier nomme la «violence secondaire» (Castoriadis-Aulagnier, 1975, p. 39 et 150), celle qui détruit l'âme et désubjectalise de façon insidieuse. Je me demande si le mot «transgressive» est pertinent pour qualifier cette violence. Quand une mère empiète à l'excès sur l'espace de son enfant ou qu'elle ne l'investit pas, quand une foule stigmatise l'étranger, l'interdit de meurtre à proprement parler n'est certes pas transgressé au sens juridique de la loi. Pourtant, l'expérience analytique nous enseigne comment ces petits meurtres d'âme sont de nature transgressive, au même titre que certaines proximités entre parents et enfants sont de nature incestuelle, c'est-à-dire des équivalents d'inceste au plan fantasmatique. Peut-être devrions-nous les nommer des violences meurtrielles. Mon choix s'est arrêté sur cinq exemples succincts. 


\section{Violence sans nom}

C'est à la suite d'une banale contrariété que tout est arrivé. Madame B., âgée de 70 ans, explose de colère et sent monter en elle cette "violence sans nom » qui l'avait si souvent envahie autrefois. Elle se déchaîne contre sa petite-fille âgée de 7 ans qu'elle avait en garde pour l'après-midi, mais aussi contre elle-même. L'expression qu'elle venait d'employer - «violence sans nom»- me surprenait, d'autant plus que j'étais en train de lire l'article de Walter Benjamin intitulé Critique de la violence (Benjamin, 2000, p. 210-240) qui parle d'une "violence pure», une violence «divine, révolutionnaire», une «violence anomique», dans laquelle la société ne respecte plus les normes de droit. Cet article est considéré comme une réponse à l'ouvrage La dictature de Carl Schmitt publié la même année en 1921 où ce dernier oppose d'un côté la "dictature des commissaires » qui apparaît en situation de crise politique - c'est-à-dire de tumultus - et qui repose sur la prescription légale d'un état d'exception, lequel est caractérisé par la levée provisoire des règles de droit - le justitium -, et de l'autre côté la «dictature souveraine» dans laquelle toutes les normes de droit ont perdu leur «force de loi» au profit d'actes et de décisions émanant du souverain dictateur qui prennent cette «force de loi». Benjamin introduit une troisième catégorie de violence, non plus celle qui fonde le droit, ni celle qui le conserve, mais celle qui le détruit, et qui est, comme le précise Giorgio Agamben, «une violence qui dépose le droit» (Agamben, 2003, p. 92). Je me demandais alors si la violence transgressive, celle que nous évoquions à l'instant, ne rejoignait pas cette notion philosophique et quasi juridique de «violence pure», de "violence sans nom».

- Une violence sans nom?

- Oui, j'ai incendié ma petite fille de mots terribles. Et je l'ai accusée de vouloir se débarrasser de moi et de désirer me jeter dans la poubelle.

Vint ensuite la démesure des auto-reproches dont elle s'accusait: elle n'était qu'un déchet dont il fallait se débarrasser, ce qui la conduisit à vouloir en finir avec la vie et provoqua son hospitalisation en psychiatrie. Madame B. est émouvante et très digne dans sa détresse. Le profond désespoir qui l'envahit prend une connotation mélancolique, avec une auto-accusation et une auto-dépréciation sur lesquelles je craignais n’avoir que peu de prise. Je craignais que la très probable violence destructrice qu'elle retournait contre elle de façon régressive devînt envahissante et par conséquent inaccessible aux interprétations.

Madame B insiste pour me dire que les symptômes qui avaient justifié ses trois précédentes analyses sont désormais bien plus terribles. Ils l'avaient 
tant fait souffrir, sa vie durant. Cela avait commencé par une voracité ininterrompue qui l'avait submergée subitement à l'âge de seize ans, alors qu'elle venait d'obtenir brillamment le baccalauréat avec mention «très bien». Elle prit 30 kilos en un seul mois. Puis ce fut des addictions à l'alcool, aux drogues et surtout aux amphétamines - elle prenait jusqu'à trente comprimés par jour -, addictions qui l'avaient conduite à des actes de tromperie, de mensonges, de vols, de délinquance légère, mais aussi à des tentatives de suicide et des hospitalisations en psychiatrie. Elle ne veut plus revivre ces cauchemars qu'elle considère, avec un réel effroi, comme le «mal absolu». Son expression, «mal absolu», m'arrête de nouveau, d'autant que Madame B. est aussi une victime indirecte de la barbarie nazie. Elle est née au début de la guerre. Ses parents avaient fui avec elle et sa sœur, de six ans son aînée, jusqu'à Lyon, et avaient franchi clandestinement la frontière suisse pour y trouver refuge durant les trois dernières années de la guerre. Du côté maternel, la famille nombreuse périt entièrement lors de la fusillade massive de leur village dans les montagnes des Carpates. Du côté paternel, toute la famille disparut au camp d'Auschwitz. Curieusement, jamais Madame B. n'évoquera la barbarie des hommes, leur brutalité; jamais elle ne parlera d'un «mal absolu» à leur sujet. Le déplacement de la notion de «mal absolu» du champ de la barbarie collective à celui d'un banal trouble addictif était pour moi assez inattendu, aussi inattendu que son emploi de l'expression «violence sans nom». Ces mots appartenaient à un autre champ sémantique. Qu'y avait-il d’à ce point terrifiant dans les actes de compulsion, et en quoi portaient-ils la marque du mal absolu? Qu'est-ce qui lui avait été transmis d'un mal absolu, de la grande histoire à la petite histoire? Madame B. serait-elle sur le point, après trois longues analyses et une guérison effective des symptômes les plus bruyants, de se heurter à une nouvelle dimension de violence en elle-même?

Madame B. raconte un rêve de trains qui lui inspire les trains de la mort qui ont emporté sa famille. Elle associe sur sa propre violence destructrice et sur sa nature profonde: on lui a tellement dit qu'elle était une enfant très méchante, une véritable enragée, qui insultait, frappait, mordait! Ce rêve dévoile aussi le degré de violence qu'elle ressent dans la séance, et qui la condamnerait au train de la mort. Madame B. a conscience d'être envahie par moments par une «violence sans nom» qui annihile tout sur son passage, et qu'elle semble retourner assez vite contre elle. Les crises de boulimie avaient été destructrices de liens et destructrices de son propre moi. Une faim de tout détruire s'ajoutait à la voracité illimitée. Elle raconte comment dans le train qui les avait amenés jusqu’à Lyon, alors qu'elle n'était qu’un bébé, 
elle avait tant hurlé durant tout le trajet, collée contre sa mère, que les officiers les ignorèrent au moment du contrôle. À Lyon, ses parents durent se terrer. La nuit, son père dormait une hache à la main. L'exode se poursuivit en Suisse. La nuit, le long des sentiers clandestins, Madame B. reste agrippée à sa mère et personne ne réussit à les séparer. Elle me signale que dans le laissez-passer suisse que la famille a conservé, elle a remarqué la mention "Mutter mit Kind», «mère avec enfant», et non «Mutter mit Kinder", «mère avec enfants». L'absence de pluriel l'intrigue et confirme l'idée qu'elle seule comptait pour sa mère. Arrivés en Suisse et pendant les trois années d'exil, le père part en camp de travail, la fille aînée est placée en internat, et la mère travaille dans une maison d'enfants comme femme de ménage. Quant au bébé à peine âgé de 1 an et demi, il reste collé à sa mère pendant toute cette période dans une relation exclusive, sur fond d'angoisse et de violence traumatique.

Madame B. est restée toute sa vie attachée à sa mère par un lien quasi sacré, une mère pourtant indisponible psychiquement à son enfant et qui exclura de façon catégorique la moindre intervention du père pourtant affectueux, attentionné et présent. Pendant la fuite vers Lyon, la mère de Madame B. attendait un troisième enfant. Elle ne voulait pas que ce bébé compromette leur seule chance de survie. Elle espérait qu'il meure vite dès la naissance, sauf si c'était un garçon. Une petite fille naquit et mourut très vite. Madame B. raconte ce drame comme un fait parmi d'autres, lié à l'exode. Elle associe alors sur une autre histoire tout aussi violente, tout aussi factuelle, celle de sa propre mère. Celle-ci, dernière d'une fratrie de six, était destinée à mourir à la naissance, du fait de la trop grande misère à cette époque dans les petits villages ukrainiens, au tout début de la Grande Guerre. La grandmère de Madame $\mathrm{B}$. avait pris la décision d'étouffer ce sixième enfant avec un coussin sitôt après la naissance. L'enfant ne dut la vie qu'à l'intervention énergique de sa plus grande sœur, l'aînée de la fratrie qui prit la décision de sauver le bébé et de l'élever elle-même. Je m’interrogeais sur ce fait choquant que la vie perd bien vite son caractère sacré lorsqu'elle est confrontée aux urgences de la vie. Madame B. est certes affectée par toutes ces histoires, non pour le tragique qu'elles recèlent, mais pour leur part impensable. Elle a toujours perçu dans sa propre vie une collusion intime entre la vie et la mort. Elle se souvient d'un troisième récit tout aussi incompréhensible: la sœur aînée de sa mère se marie, et sitôt après la naissance de son premier bébé, décide d'émigrer en Europe pour quitter la misère du village. Fait surprenant, elle emmène non pas son bébé qu'elle confie plutôt à sa famille, mais sa petite sœur qu'elle adopte et qui porte le nom du jeune couple. 
Certes, la grande histoire, celle de la guerre, des persécutions, de la misère, de la fuite désespérée, a généré un climat de violence traumatique. Mais je ne pense pas qu'elle soit directement responsable des intenses épisodes d'effondrement psychique dont a souffert Madame B. dans sa vie, ni des graves troubles addictifs qui l'ont poursuivie sa vie durant. J'irai même jusqu'à penser que la grande histoire a stimulé son acuité et son intelligence. Je pense en revanche qu'une violence bien plus redoutable est parvenue jusqu'à Madame B., que l'idéalisation de la mère a toutefois soigneusement voilée. Sa mère, traumatisée par la guerre, effrayée par la possible disparition de toute sa famille, héritière familiale d'un rapport à la vie assez énigmatique, va, pour apaiser ses angoisses et soigner sa détresse, sacrifier son enfant, ne pas l'investir, l'utiliser comme un objet de réconfort, de survie, qui lui appartient. La surestimation du réel dévouement de la mère efface la charge destructrice et les vœux de mort qui ont circulé entre elles. N'est-il pas déstabilisant de comparer cette violence-là, celle emprisonnée dans la relation envahissante et impossible d'amour entre mère et fille, et la violence de la guerre, ou celle des terrorismes? Pourtant il s'agit bien d'une violence transgressive, meurtrière d'âme, aux effets destructeurs sur la psyché, une violence meurtrielle.

\section{Violences extrêmes}

Le Centre hospitalier de Saint-Malo prend contact avec La Maison Bleue de Fougères en Bretagne (hôpital de jour et unité ambulatoire de pédopsychiatrie dont je suis le psychiatre directeur), afin que soient donnés en urgence des soins sur la personne de Killyann, un jeune garçon de 3 ans et demi, qui présente à l'école, au centre de loisirs, au foyer d'hébergement et dans sa nouvelle famille d'accueil des troubles du comportement à type de «violence extrême» qui inquiètent, découragent et empêchent de penser.

Une mesure d'aide éducative était jusqu'à présent exercée à son domicile maternel près de Fougères pour soutenir la mère dans sa fonction éducative. Le père, incarcéré pour actes de violence, est décédé en prison. Assez rapidement, une information préoccupante a été envoyée au procureur de la République précisant la grande insécurité affective et éducative dans laquelle se trouve cet enfant au domicile de sa mère. Placé en urgence par le juge des enfants dans une maison d'hébergement près de Saint-Malo, ce jeune garçon sera aussi l'objet d'une évaluation psychiatrique. Entre temps, la mère sera à son tour incarcérée en prison pendant six mois. À son retour, elle ne se présentera à aucun des rendez-vous médiatisés qu'elle avait pourtant elle-même fixés à son fils. 
L'expertise pédopsychiatrique du Centre hospitalier de Saint-Malo le décrit comme

un enfant gravement carencé, présentant un retard sévère de développement, sans langage, montrant des activités psychiques très pauvres au niveau de ses jeux spontanés et une intolérance majeure à la frustration. L'évaluation du niveau de développement sur l'échelle de Vineland précise un niveau de communication à 1 an 3 mois, une autonomie à 1 an 9 mois, une socialisation à 2 ans 4 mois. L'évaluation ADOS (Autism Diagnostic Observation Schedule) confirme l'impression clinique que ce jeune garçon ne présente pas de troubles autistiques caractérisés - les seuils n'étant pas atteints, ni en communication, ni en interaction sociale, ni au niveau des comportements stéréotypés. Un traitement neuroleptique à faible dose est prescrit à la sortie de l'expertise.

L'institutrice écrit son désarroi devant cet enfant extrêmement violent:

Après une matinée très éprouvante au cours de laquelle Killyann s'est déchaîné physiquement contre les autres enfants, les frappe, les mord et donne des coups de pieds et de poings à l'agent spécialisé des écoles maternelles, je vous informe qu'une fois de plus, nous ne savons plus quoi faire. Il ne tient pas plus de 5 minutes en classe actuellement et explose; on doit le sortir du groupe et se mettre à deux adultes pour gérer la crise. Nous ne pouvons pas faire appel à la famille d'accueil qui elle-même a demandé l'intervention d'une famille d'accueil relais. J'ai téléphoné à monsieur l'inspecteur d'académie de circonscription. Il m'a dit d'appeler les pompiers à la prochaine crise, de prévenir le procureur et de mobiliser les services sociaux. En réunion d'équipe de suivi de la scolarité, nous avons décidé de l'orienter vers un institut éducatif.

La famille d'accueil récemment nommée transmet sa préoccupation:

Depuis jeudi dernier, nous sommes appelés tous les jours pour venir le chercher à l'école. La situation se complique de jour en jour: il grimpe sur des grillages au détriment de sa sécurité. À la maison, il y a beaucoup de violences verbales et une forte défiance par rapport à notre autorité, mais par contre pas vraiment de mise en danger. Cordialement. 
Enfin, l'éducatrice spécialisée du service social rédige un rapport dont voici un court extrait:

Killyann montre un comportement extrêmement agressif et incohérent. Il m’insulte d'emblée, me jette ce qui lui passe par la main, me répète «tu veux pas jouer». Il me frappe. Il s'échappera à deux reprises de ma salle pour fuir dans les escaliers. Une collègue interviendra avec moi pour tenter de l'apaiser. Toute parole le fait flamber. Il se cogne. Je ne peux pas capter son regard. Ses mouvements sont désordonnés, le corps est comme désarticulé. Parfois, il a le regard «halluciné».

Après le bilan au sein de l'équipe thérapeutique de La Maison Bleue, nous décidons de soigner Killyann et de coordonner les actions de l'ensemble des partenaires (école, famille, familles d'accueil, centre d'hébergement et services social, éducatif et judiciaire). Nous proposons une séance par semaine de psychothérapie individuelle, deux séances par semaine de psychomotricité individuelle, cinq séances individuelles par semaine de référence éducative, une consultation de la famille d'accueil tous les quinze jours, une consultation de la mère si celle-ci accepte de se déplacer et, enfin, des rencontres avec les partenaires afin de soutenir l'investissement de tous. Nous n'envisagerons le travail en groupe d'enfants sous forme d'atelier thérapeutique que plus tard.

Killyann va progressivement s'apaiser, poser ses repères, accepter le cadre des rencontres thérapeutiques. La prise en considération par l'équipe soignante de ses angoisses, de ce qu'il ressent, de la réalité psychique autant que de la réalité effective familiale, sociale et scolaire, lui procure un début de relâchement. Un long chemin sera à parcourir avant la résolution de ses conflits internes.

Dans l'immédiat, il investit activement les séances. Il relâche son côté inaccessible, intouchable et inapprochable et modère considérablement la destructivité qui l'envahissait. La crainte intense du regard persécuteur des autres s'apaise. Il est moins aux aguets, moins dans l'hyper-contrôle. La violence mise en scène dans les jeux de figurines est davantage négociable: «Les loups surgissent brutalement le jour de Noël, mais la fête se poursuit; les extraterrestres volent le petit garçon, mais la mère le protège et se révolte; le requinmarteau et l'ours polaire veulent manger le petit garçon, mais la maman crocodile le protège avec ses grandes mâchoires; le chien qui s'est enfuit et perdu est retrouvé car son maître l'a appelé à haute voix autant qu'il a fallu...» 
L'apparition naissante du plaisir dans les explorations sensorimotrices est un bon indice de son évolution. Dans le jeu, Killyann accuse «l'enfant de la mort du père qui était en prison ». Il ébauche ainsi une mise au travail d'une culpabilité probablement liée à des vœux meurtriers de nature œedipienne.

Killyann tire un réel profit des figures parentales de substitution qui l'entourent et qui lui témoignent attention, intérêt et indulgence et qui contribuent à lui conférer un sentiment de valorisation et de consistance. Les contraintes du cadre thérapeutique sont relativement bien tolérées dans la mesure où en contrepartie une réelle relation thérapeutique personnalisée se noue dans la répétition et la continuité. Il faut dire que les comportements de violences extrêmes qu'il avait libérés répondaient en miroir à la violence transgressive qu'il avait reçue frontalement de la part de ses parents inconséquents, maltraitants, physiquement et psychiquement. Ces parents violents déchargeaient sans ménagement leur colère sans mesurer l'impact qu'ils avaient sur leurs enfants. Ils n'étaient pas en mesure de se préoccuper des enfants. Qui plus est, les adultes tiers chargés par les diverses institutions de réparer une carence majeure en assurant l'accueil familial, la scolarisation, l'éducation, etc. seront vite découragés par l'ampleur de la colère que l'enfant livrait, colère qu'ils mettaient sur le compte de «son incapacité à tolérer la frustration ». À la violence de l'enfant répondait là encore la violence de l'adulte qui était censé l'aider, sous forme d'accusations, punitions, exclusions, rejets, découragements, désintérêts, stigmatisations. Cette réaction en miroir sans fin s'est arrêtée, me semble-t-il, quand la violence a pu être pensée dans ses divers niveaux de causalité.

\section{Violence défensive}

La question difficile du vivre ensemble dans les petites collectivités est une occasion d'explorer cette clinique particulière. Prenons l'exemple de l'institution psychanalytique qui est peu théorisée. Deux tendances récurrentes viennent occuper le devant de la scène sur fond de violence consentie: un certain conformisme qui se répand assez vite au sein de l'institution encourage les discours convenus, favorise les préséances implicites et décourage les initiatives spontanées quand elles sont novatrices ou qu'elles s'éloignent de ce qui est attendu; et, parallèlement, une mise à l'index, silencieuse ou bruyante, à visée discriminatoire, conduit certains collègues sur la voie de l'exclusion, du repli, du découragement, voire de la démission.

Cette configuration caricaturale, à peine esquissée, montre comment les liens inter-analytiques sont fragilisés - à la fois illusoirement resserrés par 
le conformisme et à la fois rompus ou amoindris à regret par les mouvements discriminatoires. Elle révèle que les sociétés psychanalytiques, après la période de vitalité des moments fondateurs, sont durablement enfermées dans une organisation défensive (Major, 2008). Assez vite, le processus institutionnalisant fige cette vitalité, engloutissant dans l'inconscient collectif les fantasmes d'origine et la violence traumatique des moments fondateurs. Ce raidissement semble en partie lié et entretenu par les figures d'autorité de l'institution qui incarnent l'idéal fondateur et l'unité groupale.

Peut-être que l'espace politique au sein de l'institution n'est pas investi? L'espace politique étant «l'espace où la singularité de chacun peut apparaître et s'exprimer à égalité avec et parmi les autres» (Peuch-Lestrade, 2012). Pourquoi les psychanalystes, dès lors qu'ils se regroupent en institution, renoncent-ils, dans un "silence actif» à exprimer leur singularité? Pourquoi n'usent-ils pas de leur position d'égalité? La figure d'autorité serait, d'après cet auteur, à l'origine de ce silence actif librement consenti chez les psychanalystes, de la perte des singularités et de l'échec du travail du politique. Derrière chacune des figures d'autorité qui se succèdent dans les institutions, se dissimule l'ombre d'un Freud «autoritariste», tel qu'il se révéla dans les conflits avec ses disciples: Jung, Ferenczi et bien d'autres (Roustang, 1976). Mais pourquoi les générations après Freud héritent-elles de façon aussi durable de cet aspect autoritariste?

Je pense que ce côté préexistait chez Freud au plan fantasmatique avant même la découverte de l'Inconscient. Dans un travail sur «Le Rêve de l'injection faite à Irma» (Colin, 2007), j'avais montré qu'on y décelait un mythe fondateur de la naissance de la psychanalyse - mythe politique anticipant l'organisation groupale ultérieure. S'agissant de ce rêve princeps qui s'adresse à la communauté scientifique, on connaît l'interprétation de Didier Anzieu d'un fantasme de grossesse selon lequel, fécondé par les idées de son époque, Freud porterait en lui une grande œuvre à venir. Mais le rêve ajoute un point central qui n'a pas échappé à l'acuité d'un René Kaës, à savoir qu'il met en scène plusieurs personnes: une foule d'invités, et parmi eux, de nombreux médecins, le Dr M., Fliess, Otto, Ludwig, von Fleischl, ainsi que des confrères récalcitrants à ses théories. Tous ces personnages sont des confrères dévalorisés par le rêve: le Dr M., c'est-à-dire de Breuer, n'est pas comme d'habitude, il est très pâle, il boîte, il n'a pas de barbe. Fliess est incompétent, il oublie un morceau de gaze dans les cornets d'Emma; Otto fait une injection de triméthylamine avec une seringue qui n'est pas propre; Ludwig qui examine Irma la percute sans rigueur à travers les vêtements; von Fleischl 
n'a pas écouté le conseil de Freud de suivre une cure de démorphinisation et il en périra rapidement; les confrères récalcitrants sont tous des ignorants, etc. Le mythe politique serait donc le suivant: Freud est promis à un grand destin, il porte en lui une grande cuvre à venir, à condition de s'entourer d'une foule de disciples castrés, réduite au silence et prête à accroître son cuvre.

Ce mythe politique est un puissant fantasme narcissique dont nous serions les héritiers directs. Mais Freud va plus loin et nous transmet à la fin de son œuvre, dans Moïse et le monothéisme, une véritable théorie politique selon laquelle le peuple indiscipliné et faible s'organiserait autour de la figure d'autorité. Le grand homme, nous dit-il, n'est pas seulement celui qui produit une grande œuvre, c'est avant tout celui qui sait contraindre son prochain, celui qui sait imposer le renoncement pulsionnel aux autres, celui qui sait convaincre les foules et parvenir à influencer son temps. La grandeur d'un Prométhée, héros culturel qui apporte le feu aux hommes, se trouve - par un jeu de retournement dont seul Freud a le secret - dans l'acte de «pisser sur la flamme du rival» et par conséquent de contraindre le peuple au renoncement pulsionnel (Freud, 1932, p. 194).

Ainsi donc, la figure d'autorité incontestée du grand homme se double de celle idéalisée d'un père primitif régnant sur la horde sauvage. Je crois que nous héritons de cette coalescence entre ces deux figures et c'est bien sûr la face transgressive, celle du père primitif tout puissant et sans limites, qui exercera son action négative sur la vie institutionnelle. Cette face transgressive serait tacitement acceptée par la majorité silencieuse héritière d'une idéalisation qui voile et banalise les actes transgressifs. En témoigne le mythe soigneusement entretenu par les psychanalystes eux-mêmes de la naissance de la psychanalyse (Aulagnier, 1967; Filloux, 2009), mythe qui voudrait que cette naissance soit le fruit d'un acte transgressif et qui va jusqu'à soutenir que tout acte fondateur est transgressif. Je pense que ce mythe sème le trouble dans les esprits et qu'il a certainement contribué dans l'histoire de la psychanalyse française à préférer les scissions plutôt que soit affronté le désaccord.

\section{Violence régressive}

Si le fantasme d'un père primitif tout-puissant domine silencieusement l'organisation de groupes restreints, quel serait celui de la société tout entière?

La réponse apparaîtra peut-être en étudiant la violence nihiliste que génère la société dans les périodes tourmentées de son histoire. J'emprunte le mot «nihiliste» au vocabulaire des écrivains, philosophes et historiens de 
la fin du XIX ${ }^{\mathrm{e}}$ siècle et du début du Xx $\mathrm{X}^{\mathrm{e}}$ siècle. Ces auteurs se sont penchés sur la question du nihilisme culturel et politique de leur époque. J'ai volontairement évité le vocabulaire de la psychanalyse qui, à la même époque, introduit la notion de pulsion de mort, même si les notions de nihilisme et de pulsion de mort sont très voisines. Toutes deux font appel au retour à l'inanimé, au rien, au degré zéro de tension, au nihil du Nirvana, et en même temps à la destructivité la plus bruyante, à la violence la plus désintriquée. Je n'utilise pas les mots plus actuels, barbarie, terrorisme, tyrannie du mal, génocide, crime contre l'humanité, etc., car je préfère ouvrir un autre champ lexical. En fait, mon travail s'établit à distance de l'actualité, plutôt en observateur d'études des époques anciennes. Il apparaît que la violence nihiliste qui s'installe silencieusement ou bruyamment dans la vie sociale, telle qu'elle a été décrite dans les exemples classiques des nihilismes français, russe et allemand, résultait des effets délétères d'une épreuve de désillusion. L'idéalisation de l'objet et sa variante sociétale - l'illusion idéologique - contiennent et retiennent, il me semble, une charge importante de violence et de destructivité que les épreuves de désillusion et de désidéalisation libèrent. Dans le meilleur des cas, lorsque ces épreuves sont l'objet de discussion, d'élaboration analytique et de travail de culture, la libération est salutaire. Dans d'autres cas, la violence se déchaîne de façon massive et régressive, ouvrant la voie à des actes de violence terroriste irréparables, sur un mode régressif, signant par là le refus de renoncer à l'illusion.

La régression prend la forme du clivage intérieur-extérieur que Freud avait décrit pour parler de la toute première étape du développement du moi, en termes de «moi plaisir purifié» et de «monde extérieur déplaisir haï» (Freud, 1915, p. 37), ou encore de «moi purement hédonique» et de «monde extérieur étranger et menaçant» (Freud, 1930, p. 9). Pour que le moi se développe, il faut qu'il rejette à l'extérieur le négatif éprouvé, source de déplaisir, d'angoisse et de désillusion. Pour qu'il puise en lui-même suffisamment de force, le moi doit s'identifier à un idéal positif, il doit croire en une puissante illusion qui lui procurera satisfaction et réassurance, un idéal suffisamment purifié de toutes sortes de scories pour engendrer un sentiment d'unité naissante et de certitude d'exister. Lors d'un mouvement régressif, le moi idéal infantile (l'ancien moi plaisir purifié) détrône et neutralise l'idéal du moi qui ne joue plus son rôle de guide éclairant les valeurs culturelles les plus hautes. L'idéal du moi abdiquerait face à un moi anéanti par la désillusion, et donnerait libre cours au moi idéal par essence tout puissant en proclamant l'état d'exception. 
La violence pure libérée reflète alors l'extériorisation d'un inconscient d'autant plus puissant qu'il se trouvait enserré dans les mailles de l'idéalisation.

Pourquoi ce retour si prompt à l'objectivation déshumanisante? Certes, on pensera que, afin de mieux réparer le traumatisme de la désillusion et de la destructivité reçue, la société puise dans ses sources les plus lointaines la substance vitale narcissique qui fait provisoirement défaut. On pensera surtout qu'elle cherche à retrouver les conditions premières pour générer une nouvelle illusion, pour répéter et agir l'idéalisation. Tout au mieux, on espérera que sera entamé un travail de perlaboration sur les mythes d'origine et la violence traumatique des moments fondateurs à l'origine de l'illusion (Colin, 2011, p. 263).

La violence régressive serait un retour vers les temps d'origine. Elle servirait la cause transgressive de répéter le piège de l'idéalisation première en se donnant la possibilité de créer de nouvelles illusions. Si, paradoxalement, elle est l'occasion de réinterroger les sources premières de l'illusion ainsi que la violence qui a été piégée dès l'origine, elle n'en reste pas moins meurtrielle, c'est-à-dire source vive de destruction. La conquête coloniale par exemple n'est pas seulement l'acquisition d'un nouveau territoire et de nouvelles richesses, conférant un accroissement de puissance au profit de l'envahisseur. Elle a imposé aux peuples, sous couvert d'un partage culturel idéalisé, un mépris et un assujettissement. Peut-on s'étonner, plusieurs générations après la décolonisation, que la violence surgisse en retour?

\section{Violence culturelle}

À une époque où de nouveaux pouvoirs conquérants diffusent par les médias des scènes cruelles de décapitation dans le but d'affirmer leur hégémonie, d'afficher leur idéologie et de terroriser l'ennemi, il n'est pas inutile, au lieu de clore le débat par une réaction affective d'indignation justifiée, de se souvenir que le vieil Occident avait en toute légalité, sur la place publique, décapité, écartelé, écorché vif, pendu haut et court, guillotiné, fusillé, etc.

Quand doit-on parler de cruauté barbare, de «violence pure» qui dissout le droit? Quand parlerons-nous de violence qui fonde le droit, le conserve? Où se situe la limite?

Dans les œuvres de cultures, littéraires, philosophiques ou artistiques, il arrive que cette limite s'estompe imperceptiblement, semant le trouble dans les repères identificatoires. Je reprendrai volontiers l'exemple du Caravage que j'avais développé à Naples (Colin, 2014) pour montrer aujourd'hui en 
quoi des violences meurtrielles peuvent se dissimuler dans les œuvres et agir sur nos représentations mythiques.

Le Caravage a peint des scènes bibliques portant sur l'éradication de la violence. Dans le tableau Judith décapitant Holopherne, le mal est représenté par Holopherne, un tyran assyrien qui semait la mort et la terreur dans les terres qu'il venait de conquérir. On peut penser assurément que Le Caravage, à l'apogée de son art, fait œuvre de culture en peignant le geste politique courageux de l'héroïne tuant le tyran. L'œuvre d'art contribue à ce travail d'élaboration des mythes fondateurs que nous évoquions précédemment. Toutefois, notons que la scène qu'il nous donne à voir est particulièrement brutale. Montre-t-elle dans sa juste crudité la brutalité nécessaire à tout acte qui fonde le droit, ou cherche-t-elle à partager avec le spectateur une pointe d'excitation provoquée par l'horreur de l'acte meurtrier, excitation qui détournerait un instant le spectateur du travail de pensée ou d'association?

Nous pourrions nous poser la même question devant le tableau tout aussi violent d'Artémisia Gentileschi traitant du même sujet, d'autant que nous savons que cette remarquable femme peintre a été victime très jeune

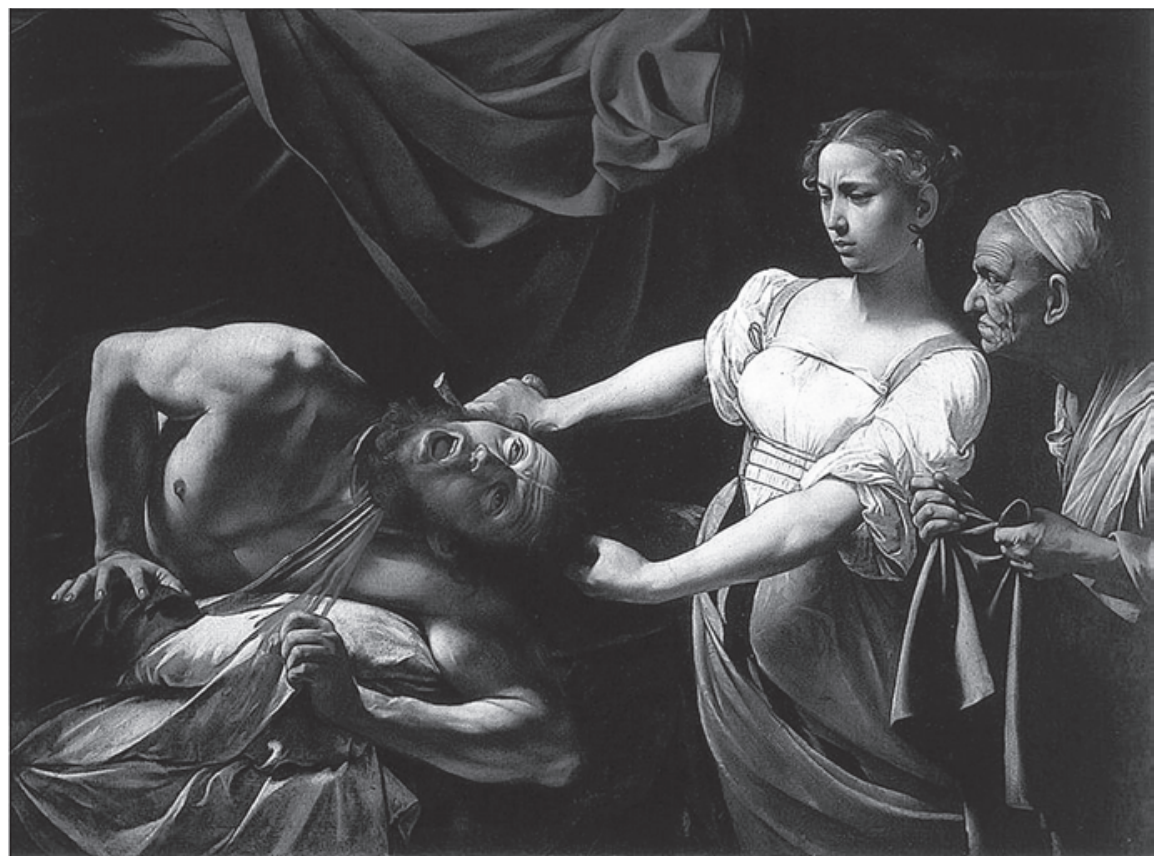

Le Caravage, Judith décapitant Holopherne, 1598-1599.

Galerie nationale d'art ancien, Rome. 
d'un viol. On suppose que cette peinture contribua à libérer une violence destructrice en elle. Quelles que soient les raisons de cette mise en scène cruelle, ces deux tableaux restent des moments culturels forts.

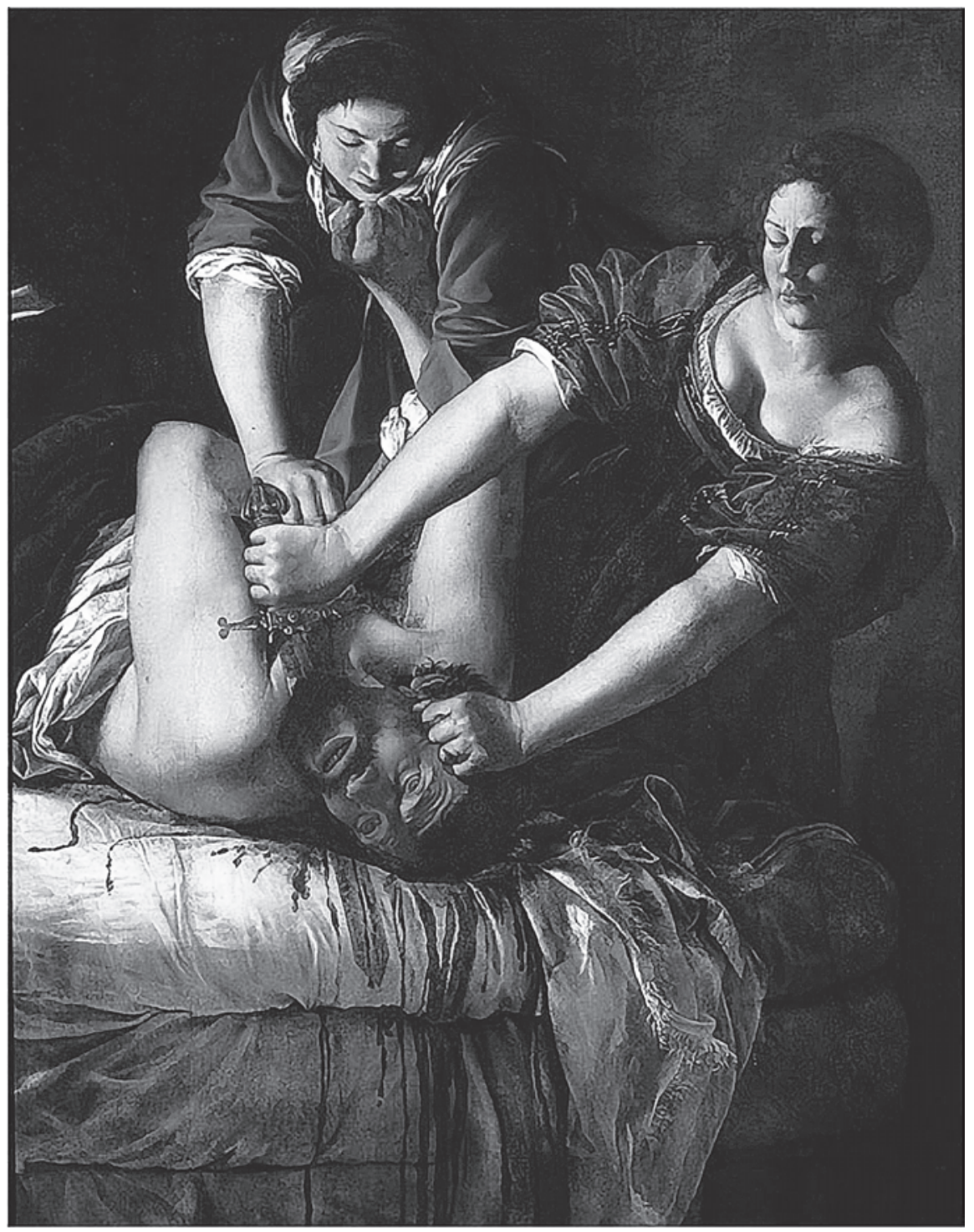

Artémisia Gentileschi, Judith décapitant Holopherne, 1618-1620. Musée des Offices, Florence. 
Douze ans plus tard, à Naples, Le Caravage est un homme en fuite qui vient d'être violemment agressé et laissé pour mort au coin d'une rue. Il peint une deuxième version du David, où il met en scène un jeune berger

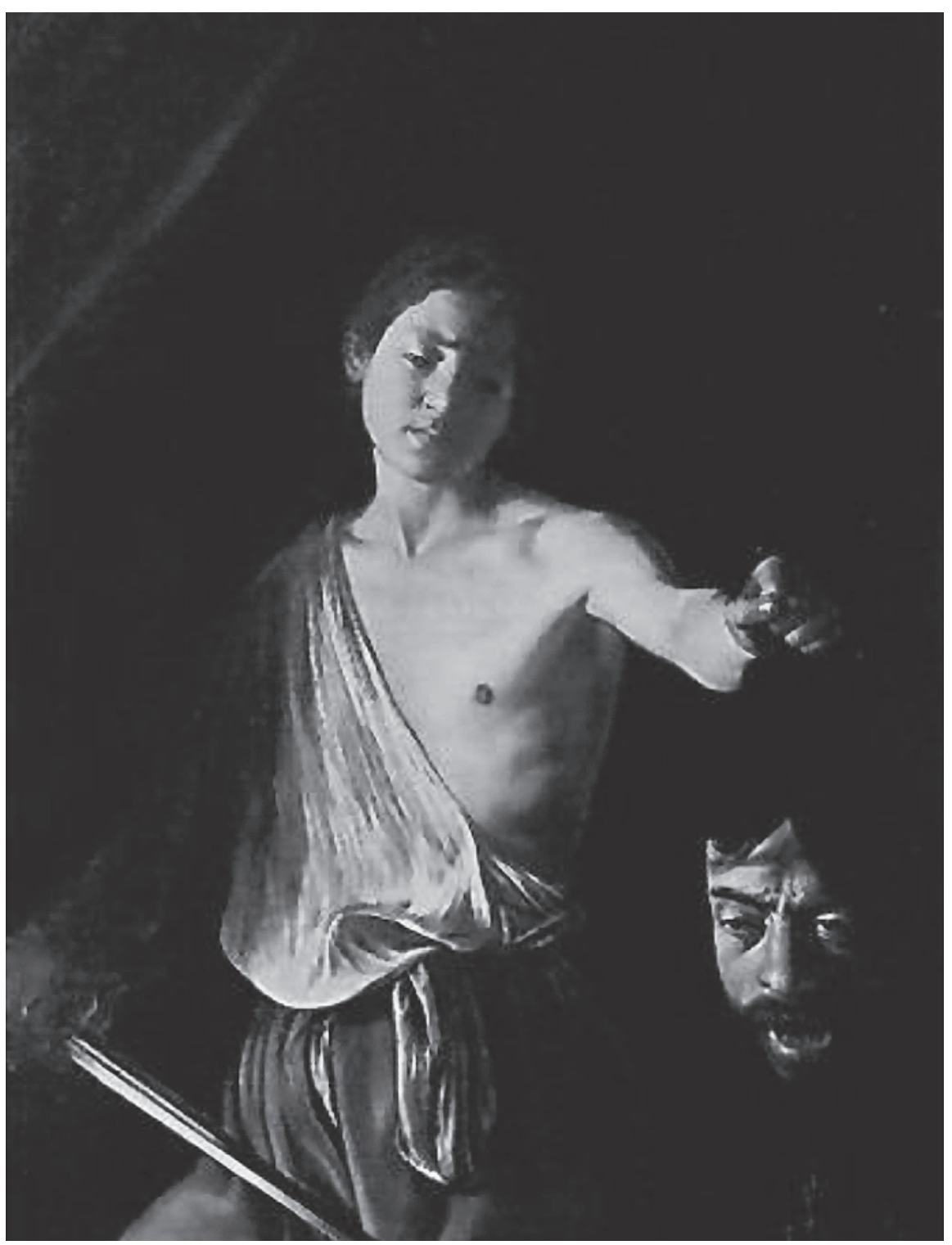

Le Caravage, David tenant la tête de Goliath, 1606-1607.

Galerie Borghèse, Rome. 
courageux qui a débarrassé son peuple de la barbarie sauvage de Goliath. Mais, cette fois-ci, point de complaisance ni d'excitation à partager avec le spectateur. Il peint un David plein d'humilité et de retenue, donnant à voir le sort réservé à celui qui semait la terreur. «L'humilité tue l'orgueil», est-il écrit en abréviations sur l'épée du berger («H-AS OS»; Humilitas occidit superbiam). Dans ce tableau, Le Caravage interroge le rapport intime qu'il entretient lui-même avec la cruauté en choisissant de peindre son propre visage en Goliath décapité.

Nous mesurons toute la puissance d'évocation de ces trois scènes étonnantes, très proches l'une de l'autre, d'égale importance en matière de Kulturarbeit, mais qu'une épreuve de désidéalisation semble pourtant séparer, levant le voile sur ce que la loi de l'interdit de meurtre délimite. N'est-il pas audacieux dans cet exemple de parler de violence meurtrielle? Le Caravage et Artémisia Gentileschi n'ont-ils pas contribué dans ce court exemple à colorer imperceptiblement de leur fantasme de meurtre respectif les mythes culturels, trésor de l'inconscient collectif?

Que dire de la violence décrite dans ces cinq courtes vignettes? Je remarque tout d'abord qu'il y a la violence perceptible au premier plan, celle qui éclate, ici, sous nos yeux, qui est spectaculaire, qui dérange, qui fascine, qui pousse à consulter, qui pousse à réagir en miroir, qui pousse à mettre en œuvre des actions immédiates, éducatives, sociales, politiques, culturelles. Et puis, il y a là, cachée derrière, au second plan, quasiment invisible, quasiment silencieuse, une autre violence discrète, piégée, immobile, mais dont les effets à distance sont considérables. C'est cette violence qui m'apparaît destructrice, désubjectivante, responsable de meurtres d'âme dont nous parlions en introduction: une violence meurtrielle qui laisse toujours des traces. Elle n'est pas comparable à la violence dévastatrice générée par exemple par un événement traumatique. Car les effets d'un traumatisme sont plutôt du côté de l'intensité, de l'économie psychique, du débordement des pareexcitations et ils renvoient à la capacité ou non pour la victime d'absorber l'événement, d'élaborer une représentation. La violence meurtrielle est tout autre. Elle contient un puissant désir d'annihilation. Elle ampute une part importante de la subjectivité.

Robert C. Colin robertcolin99@gmail.com 


\section{Bibliographie}

Aulagnier, P. (1967). Le «désir de savoir» dans ses rapports à la transgression. L'Inconscient, $1,109-124$.

Agamben, G. (2003). État d'exception. Homo Sacer. Paris: Seuil.

Benjamin, W. (1921). Critique de la violence. CEuvres I (2000, 210-243). Paris: Gallimard.

Castoriadis-Aulagnier, P. (1975). La violence de l'interprétation. Paris: Puf.

Colin, R.C. (2007). Le rêve de «l'injection faite à Irma» et le mythe biblique de la création de la psychanalyse. Le Coq-Héron, 191, 65-76.

Colin, R.C. (2007). La violence nihiliste. Topique, 99, 139-171.

Colin, R.C. (2011). Nihilisme et régression. Dans Lévy G. (dir), L'esprit d’insoumission. Réflexions autour de la pensée de Nathalie Zaltzman. Paris: Campagne Première.

Colin, R.C. (2014). La regressione collettiva, Dans De Rosa B. (dir.), A cura di Barbara De Rosa. Il male dal prisma del Kulturarbeit. Sull'opera di Nathalie Zaltzman (p. 86-102). Milan: Franco Angeli.

Filloux, J. (2009). La transgression de la psychanalyse et dans la psychanalyse. Topique 106, 34-48.

Freud, S. (1915). Pulsions et destin des pulsions. Métapsychologie. Paris: Gallimard, 1968.

Freud, S. (1930). Malaise dans la civilisation. Paris: Puf, 1971.

Freud, S. (1932). Sur la possession de feu. Résultats, idées et problèmes II. Paris: Puf, 1987.

Major,R.(2008).Croyanceenlacommunautéetcommunautédecroyance.Repéréà,http://www. quatrieme-groupe.org/activites-scientifiques/debats-et-tables-rondes?id=91\&annee= 2008-2009

Peuch-Lestrade, J. (2012). Autorité et tentation autoritaire? Le Coq-Héron, 208, 54-66.

Roustang, F. (1976). Un destin si funeste. Paris: De Minuit.

Schmitt, C. (1921). La dictature, traduit de l'allemand. Paris: Le Seuil, 2000.

Zaltzman, N. (1979). La pulsion anarchiste. Topique 24, 25-64.

Zaltzman, N. (1998). De la guérison psychanalytique. Paris; Puf.

Zaltzman, N. (2007). L'esprit du mal. Paris: Éditions de l'Olivier. 